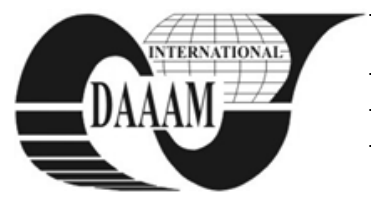

Annals of DAAAM for 2011 \& Proceedings of the 22nd International DAAAM Symposium, Volume 22, No. 1, ISSN 1726-9679 ISBN 978-3-901509-83-4, Editor B. Katalinic, Published by DAAAM International, Vienna, Austria, EU, 2011 Make Harmony between Technology and Nature, and Your Mind will Fly Free as a Bird

\title{
COMPARISON OF TRANSFER FUNCTION RATIONAL APPROXIMATIONS FOR LTI-TDS
}

PEKAR, L[ibor] \& KURECKOVA, E[va]

\begin{abstract}
Many controller design approaches for linear timeinvariant time delay systems (LTI-TDS) require a delay approximation either in the state space or that of the transfer function. This paper is focused on the preliminary comparison of some selected easy-handling rational approximations of exponential elements in LTI-TDS transfer functions. Unlike traditional concept, internal (state) delays are considered as well. A program testing interface in Matlab-Simulink environment is developed.
\end{abstract}

Key words: time delay systems, Padé approximation, Laguerre shift, Kautz shift, Fourier analysis

\section{INTRODUCTION}

In recent decades a huge number of papers and works have been focused on model reduction of rational approximation of LTI-TDS, see e.g. (Makilla \& Partington, 1999a; Makilla \& Partington, 1999b; Battle \& Miralles, 2000). A fair overview of some methods and approaches has been published in (Partington, 2004). However, a overwhelming majority of these methods deals with delays in input-output relation only ignoring state or internal delays on the left-hand side of differential equations, i.e. in the transfer function denominator (Zitek \& Vitecek, 1999; Richard, 2003).

This paper, on the other side, focuses transfer function rational approximations for systems with internal delays since there are no theoretical results about approximations convergence and accuracy, and one can expect an interesting comparative results. When methods selection, emphasis is also put on handling with them; they must be easy to deal with so that anyone can use them effortlessly. The common principle of all approaches consists in substitution of exponential terms in the transfer function with rational fraction in Laplace complex variable $s$. Approaches are compared via $H_{\infty}$ and $H_{2}$ norms.

For the authors intentions, the obtained results can be utilized e.g. when computer digital implementation of so called anisochronic controller obtained via algebraic controller design (Pekar \& Prokop, 2008).

A Matlab-Simulink interface for testing all the applied methods is developed as well to make the comparison easier.

\section{APPROXIMATION METHODS}

In this chapter, used approaches and methods are described.

\subsection{Padé Approximation}

One of the most favorite and used rational approximations worldwide is the Padé one (Partington, 2004) given by the relation

$$
\exp (-s T) \approx \frac{P(-s)}{P(s)}, P(s)=\sum_{k=0}^{n}\left(\begin{array}{l}
n \\
k
\end{array}\right) \frac{(2 n-k) !}{(2 n) !}(s T)^{k}
$$

\subsection{Diagonal Padé Approximation}

Diagonal Padé approximant reads (Battle \& Miralles, 2000; Richard, 2003)

$$
\exp (-s T) \approx \frac{P(s)}{P(-s)}, P(s)=\sum_{k=0}^{n} \frac{(2 n-k) !}{k !(n-k) !}(-s T)^{k}
$$

One can, however, verify that (1) and (2) give the same resulting form.

\subsection{Shift Operator Approximations}

These methods are based on the fact that a delay term $\exp (-s T)$ expresses a shift operator and can be subjected to MacLaurin series expansion. A brief description of three the most important shift operators follows.

Laguerre shift. The eventual relation for this shift operator approximant reads

$$
\exp (-s T)=\lim _{n \rightarrow \infty}\left(\frac{1-\frac{s T}{2 n}}{1+\frac{s T}{2 n}}\right)^{n}
$$

see e.g. (Makilla \& Partington, 1999a).

Kautz shift. According to (Makilla \& Partington, 1999a), the Kautz shift of the $n$th order is given by

$$
\exp (-s T)=\lim _{n \rightarrow \infty}\left(\frac{1-\frac{s T}{2 n}+\frac{1}{2}\left(\frac{s T}{2 n}\right)^{2}}{1+\frac{s T}{2 n}+\frac{1}{2}\left(\frac{s T}{2 n}\right)^{2}}\right)^{n}
$$

For input-output delays only, Kautz shift is asymptotically (approximately) twice more accurate than the Laguerre one.

Padé shift. This type of shift approximation proceeds from the second order Padé approximation and can be expressed as

$$
\exp (-s T)=\lim _{n \rightarrow \infty}\left(\frac{1-\frac{s T}{2 n}+\frac{1}{3}\left(\frac{s T}{2 n}\right)^{2}}{1+\frac{s T}{2 n}+\frac{1}{3}\left(\frac{s T}{2 n}\right)^{2}}\right)^{n}
$$

\subsection{Fourier Analysis Based Method}

This type of approximation was obtained from the analysis of the delay-in-feedback step response by the Fourier series expansion of the periodic part of the response. Using a negative feedback, one can obtain the following formula (Battle \& Miralles, 2000)

$$
\begin{aligned}
& \exp (-s T)=\frac{W(s)}{1-W(s)} \\
& W(s)=\frac{1}{2}-\frac{1}{4} T s+\frac{2}{\pi^{2}} T^{3} s^{3} \sum_{k=0}^{n} \frac{1}{(2 n+1)^{2}} \frac{1}{s^{2} T^{2}+(2 n+1) \pi}
\end{aligned}
$$




\section{APPROXIMATION MEASUREMENT}

Transfer function norms instead of any complex analytic method for the comparison of approximation accuracy are used in this paper since this conception is sufficient for particular cases and for engineering practice.

\section{1 $\mathrm{H}_{2}$ Norm}

The quadratic or $\mathrm{H}_{2}$ norm of a stable strictly proper transfer function $G(s)$ reads

$$
\|G\|_{2}=\sqrt{\frac{1}{2 \pi} \int_{-\infty}^{\infty}|G(\mathrm{j} \omega)|^{2} \mathrm{~d} \omega}
$$

\section{2 $H_{\infty}$ Norm}

This norm expresses so called $L_{2}$ gain and can be calculated as the supreme of the amplitude frequency characteristics

$$
\|G\|_{2}=\sup _{\omega}|G(\mathrm{j} \omega)|
$$

In (7) and (8) $G(s)$ is taken as the difference between the nominal and approximated transfer functions.

\section{PROGRAM IMPLEMENTATION}

All the methods described above have been implemented in a Matlab-Simulink program module with a user-friendly interface. The program enables a user to insert nominal $G(s)$, select approximation methods with appropriate orders and choose a norm or norms. It returns a graphical comparison of all chosen amplitude frequency responses, a table of calculated norms and, in the text form, we can obtain approximated transfer functions.

Due to the limited space, no graphics of the program interface are given here.

\section{STUDY CASE}

As mentioned above, the authors intent to use the comparison when selection of a suitable rational approximation of anisochronic (i.e. those with internal delays) controllers. This is one of possible steps of controllers' digitalization for the computer implementation.

Consider for example a first order unstable LTI-TDS

$$
G_{S}(s)=\frac{0.6}{s-0.2 \exp (-0.8 s)} \exp (-4 s)
$$

can be controlled by the controller of the transfer function

$$
G_{R}(s)=\frac{3.78 s+0.9-0.33 \exp (-0.8 s)}{s+2(1-\exp (-4 s))}
$$

see details in (Prokop \& Pekar, 2010).

Let $n \in\{1,2,3,4,5\}$ and test the accuracies for all methods introduced above. The best results for each of them are displayed in Tab 1.

\begin{tabular}{|c|c|c|}
\hline Method & $H_{\infty}$ & $n$ \\
\hline Padé approx. & 0.729 & 1 \\
\hline Laguerre shift & 0.729 & 1 \\
\hline Kautz shift & 0.694 & 4 \\
\hline Padé shift & 0.82 & 1 \\
\hline Fourier analysis & 1.134 & 5 \\
\hline
\end{tabular}

Tab. 1. Comparison of rational approximations of (10)
Apparently, the Kautz shift of the $4^{\text {th }}$ order provides the best result for the study case; however, Padé approximation and Laguerre shift even of the $1^{\text {st }}$ order give comparable estimations which are much easier to utilize. Notice that for anisochronic systems, a higher order does not mean a better approximation in general.

$\mathrm{H}_{2}$ norm can not be utilized since for non-strictly proper transfer functions reaches infinity.

\section{CONCLUSION}

In our contribution, we have focused some easy-handling transfer function rational approximations. Dealing with internal delays in controlled plants as well as controllers was the primary motivation for our work since no theoretical results were made for this purpose.

A GUI application has been developed in Matlab for comparison and calculating approximations of the delay systems. The application allows easy entering a plant transfer function, selecting methods to be compared and the choice of norms by which the accuracy of approximation is calculated. It is also possible to specify an order of the approximation for each selected method.

The main finding is that the accuracy of an approximation is not straightly dependent on its order in all cases, as holds in the case of input-output time delay.

\section{ACKNOWLEDGEMENTS}

The authors kindly appreciate the financial support which was provided by the Ministry of Education, Youth and Sports of the Czech Republic, in the grant No. MSM 7088352102 and by the European Regional Development Fund under the project CEBIA-Tech No. CZ.1.05/2.1.00/03.0089.

\section{REFERENCES}

Battle, C. \& Miralles A. (2000). On the approximation of delay elements by feedback. Automatica, Vol. 36, Issue 5, 659664, ISSN 0005-1098

Havlena, V. (1999). Modern Control Theory (in Czech), CVUT Publishing, ISBN 80-01-02036-3, Prague

Makilla, P. M. \& Partington, J. R. (1999a). Laguerre and Kautz shift approximations of delay systems. International Journal of Control, Vol. 72, No. 10, 932-946, ISSN 00207179

Makilla, P. M. \& Partington, J. R. (1999b). Shift operator induced approximations of delay systems. SIAM Journal on Control and Optimization, Vol. 37, Issue 6, 1897-1912, ISSN 0363-0129

Partington, J. R. (2004). Some frequency-domain approaches to the model reduction of delay systems. Annual Reviews in Control, Vol. 28, Issue 1, 65-73, ISSN 1367-5788

Pekar, L. \& Prokop, R. (2008). Control of delayed integrating processes using two feedback controllers - RMS approach. Proceedings of the 7th WSEAS International Conference on systems Science and Simulation in Engineering (ICOSSSE '08) - Recent Advances in System Science and Simulation in Engineering, Venice, Italy, November 21-23, 2008, ISBN 978-960-474-027-7, pp. 3540, WSEAS, Athens, Greece

Prokop, R. \& Pekar, L (2010). Control of delay systems - A meromorphic function approach. Journal of Cybernetics and Informatics, Vol. 9, No. 1, 41-49. ISSN 1336-4774

Richard, J. P. (2003). Time-delay systems: An overview of some recent advances and open problems. Automatica, Vol. 39, Issue 10, 1667-1694, ISSN 0005-1098

Zitek, P. \& Vitecek, A. (1999). The Control Design of Subsystems with Delays and Nonlinearities (in Czech), CVUT Publishing, ISBN 80-01-01939-X, Prague 